\title{
Classifying projective modules over some
} semilocal rings

Dubrovin, Nikolay and Puninski, Gena

2006

MIMS EPrint: 2006.402

Manchester Institute for Mathematical Sciences

School of Mathematics

The University of Manchester

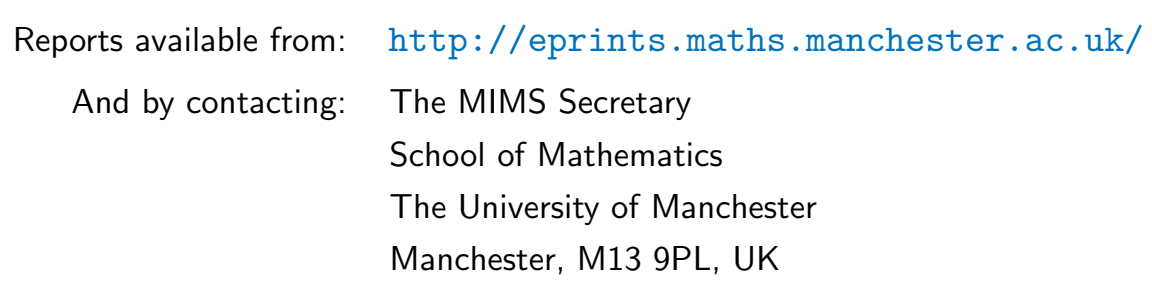

ISSN 1749-9097 


\title{
CLASSIFYING PROJECTIVE MODULES OVER SOME SEMILOCAL RINGS
}

\author{
NIKOLAY DUBROVIN AND GENA PUNINSKI
}

\begin{abstract}
We investigate the module theory of one class of semilocal rings connected with nearly simple uniserial domains. For instance, we classify finitely presented and pure-projective modules over these rings and calculate their projective dimension.
\end{abstract}

\section{INTRODUCTION}

A ring $R$ is said to be semilocal, if its factor by the Jacobson radical, $\mathrm{J}(R)$, is a semisimple artinian ring. For instance, every semilocal ring has only finitely many simple modules $S_{1}, \ldots, S_{n}$. If $M$ is an $R$ module, then $M / M \mathrm{~J}(R)$ is a direct sum of copies of the $S_{i}: M / M \mathrm{~J}(R) \cong$ $S_{1}^{\left(\alpha_{1}\right)} \oplus \ldots \oplus S_{n}^{\left(\alpha_{n}\right)}$, where $\alpha_{i}$ are cardinals. We call the tuple $\left(\alpha_{1}, \ldots, \alpha_{n}\right)$ the dimension vector of $M, \operatorname{dim}(M)$. If $P$ is finitely generated projective, then $P$ is projective cover of $P / \mathrm{J}(P)$, hence $P$ is uniquely determined by its dimension vector. The set of all tuples $\operatorname{dim}(P)$, where $P$ runs over finitely generated projective $R$-modules, is a subsemigroup of $\mathbb{N}^{n}$ called a finite projective spectrum of $R$. Facchini and Herbera [6] proved that finite projective spectra of semilocal rings can be described as semigroups $G \cap \mathbb{N}^{n}$ with order units, where $G$ is a subgroup of $\mathbb{Z}^{n}$. It may be difficult to calculate the finite projective spectrum of a particular semilocal ring. But as soon as this task is completed, we know finitely generated projective modules and their decomposition theory.

A similar problem for infinite projective spectra (that is, for dimension vectors of non-finitely generated projective modules) of semilocal rings is very much open. But there is a recent breakthrough in this area due to Př́hoda [17]. It follows from his results that over a semilocal ring every

2000 Mathematics Subject Classification. 16D40, 16D70, 16E10, 16 S50.

Key words and phrases. Semilocal ring, uniserial module, projective module, pureprojective module, global dimension.

The first author is supported by the EPSRC grant no. EP/D077907/1. He also thanks Manchester University for kind hospitality.

The second author is partially supported by the NSF grant no. DMS-0612720. 
projective module (finitely generated or not) is uniquely determined by its dimension. Thus to classify projective modules over semilocal rings is the same as to describe their (finite and infinite) projective spectra, that is, the collection of all possible dimension vectors of projective modules.

Of course the problem reduces to the finitely generated case, if every projective module over a semilocal ring $R$ is a direct sum of finitely generated (projective) modules. For instance, this is the case when $R$ is commutative (see [9]) or semiperfect (see [13, Cor. 24.14]). A more detailed treatment of this situation can be found in [15]. Although this is not true in general, the examples of semilocal rings, where not every projective is a direct sum of finitely generated modules, are quite rare, and the calculations of projective spectra for these examples is usually an arduous task.

For instance, using universal localizations Gerasimov and Sakhaev [8] gave an example of a semilocal ring with two simples and a non-finitely generated projective module $P$ whose dimension is $(1,0)$, hence $P / J(P)$ is cyclic. Even for this example the classification of projective modules has not been completed yet.

In [19] Puninski constructed an example of a semilocal ring $S$ with two maximal ideals and classified all projective modules over $S$. Namely, every finitely generated projective $S$-module is free (and has $(n, n)$ as a dimension), but the projective spectra of $S$ is a cone $\{(\alpha, \beta) \mid \alpha \geq \beta\}$. For instance (as in the aforementioned Gerasimov-Sakhaev example) there exists a non-finitely generated projective $S$-module $V$ of dimension $(1,0)$, and every projective $S$-module is a direct sum of copies of $S$ and $V$.

A lot of information about ring theoretic properties of $S$ can be derived from [20]. For instance, $S$ is (left and right) distributive, uniform, and the lattice of one-sided ideals of $S$ is also known. The ultimate goal of this paper is to classify pure-projective modules over $S$. As we will see below this amounts to classification of projective modules over a semilocal ring $S^{\prime}=\operatorname{End}(S \oplus I)$, where $I$ is an ideal of $S$ consisting of non-monomorphisms. We will show that $S^{\prime}$ has exactly three maximal two-sided ideals and prove that every projective $S^{\prime}$-module is a direct sum of copies of 4 indecomposable projectives (two of them are finitely generated and two are not). For instance, the projective spectrum of $S^{\prime}$ consists of vectors $(\alpha+\beta, \gamma+\delta, \alpha+\gamma)$, where $\alpha, \beta, \gamma$ and $\delta$ are cardinals. The proof of this result demonstrates an extreme effectiveness of Př́hoda's theorem. Namely, after some (quite technical) calculations we arrive on the list of indecomposable projective 
$S^{\prime}$-modules which is apparently complete. After that an application of Př́hoda's result reduces proofs to some cardinal arithmetic.

The ring $S$ was constructed in [19] as the endomorphism ring of a (finitely presented) module $R / r R$, where $R$ is a nearly simple uniserial domain, and $r$ is (any) nonzero element of $\mathrm{J}(R)$. Here a uniserial domain is said to be nearly simple, if $\mathrm{J}(R)$ is the only nonzero two-sided ideal of $R$. From point of view of finitely presented modules nearly simple uniserial domains are very alike to rings of finite representation type. Indeed (see [18, Ch. 14]) every finitely presented $R$-module is a direct sum of copies of $R$ and $R / r R$, $0 \neq r \in \mathrm{J}(R)$, and all the modules $R / r R$ are isomorphic.

If $T$ is a ring of finite representation type, $N$ is a sum of all indecomposable $T$-modules (one for each isomorphism type) and $U=\operatorname{End}(N)$, then Auslander's result (see [1, Sect. VI.5]) says that $U$ is of finite representation type again. We prove that $S$ inherits a similar property from its predecessor $R$ : every finitely presented (right) $S$-module is a direct sum of copies of $S$, $S / I$ and $I$.

Note that the examples of nearly simple uniserial domains are also rare. The one we know was constructed by Dubrovin [4] to give a concise example of a simple radical ring. However, the elements of this example are highly noncommutative fractions of elements of a group ring, and the calculations with them is often a nontrivial task. So, at is was pointed out by Ivo Herzog, this ring $S$ behaves like a category of functors, or as a shrinking of a tensor ring (see Wisbauer [21, Ch. 10]). To confirm this point of view we prove that $S$ is coherent and has global dimension 2. Furthermore, if $R$ is the aforementioned example of a nearly simple uniserial domain, then every ideal of $S$ is countably generated. Thus this is a good illustration to some results of Jensen [10].

We succeeded in calculations in $S$ by having chosen a pair $f, g \in S$ such that $\operatorname{ker}(g)=\operatorname{im}(f)$, where $g$ is an epimorphism and $f$ is a monomorphism, as a vague 'basis' for $S$. After that (and implying some mild model theory) the calculations go quite smoothly. We will illustrate our 'abstract' considerations by examples of 'real' calculations in $R$ and $S$. Although far from being easy, in these examples, the choice of $f$ and $g$ can be made precise.

On the other hand $S$ clearly resembles the Gerasimov-Sakhaev example $R_{\Sigma}$. Thus there is a hope to use $S$ to render calculations in the universal localization $R_{\Sigma}$, but this is beyond the scope of this paper. 


\section{UNISERIAL DOMAINS}

A module $M$ is said to be uniserial, if the lattice of submodules of $M$ is a chain. A ring $R$ is right (left) uniserial, if $R_{R}\left({ }_{R} R\right)$ is a uniserial module. $R$ is a uniserial ring, if $R$ is right and left uniserial.

Thus, if $R$ is a (right) uniserial ring and $a, b \in R$, then there are $s, t \in R$ such that either $a s=b$ or $b=a t$. For instance, the localization of the ring of integers $\mathbb{Z}$ with respect to a prime ideal $p \mathbb{Z}$ is a uniserial ring. This ring is commutative and noetherian. However the examples we consider below are very far from having these properties. Every uniserial ring is local: the Jacobson radical of $R, \mathrm{~J}(R)$, is a unique maximal right and left ideal of $R$. It follows that every element from $R \backslash \mathrm{J}(R)$ is invertible, hence $\mathrm{U}(R)=R \backslash \mathrm{J}(R)$ is the group of units of $R$. Thus, if $a, b$ are elements of a (right) uniserial ring $R$ and $a \notin b R$, then $b=a s$ for some $s \in \mathrm{J}(R)$. Furthermore $R / \mathrm{J}(R)$ is a skew field, and $R$ is a skew field iff $\mathrm{J}(R)=0$.

A ring $R$ is a domain, if $R$ has no zero divisors. If $R$ is a uniserial domain, it is Ore, hence has a classical quotient (skew) field $D$. Since $R$ is a uniserial order in $D$, for every $0 \neq d \in D$, either $d \in R$ or $d^{-1} \in R$ holds. Furthermore, if $d \in D \backslash R$, then $d^{-1} \in \mathrm{J}(R)$.

Let $R$ be a uniserial domain, $0 \neq r \in \mathrm{J}(R), M=R / r R$, and $S=\operatorname{End}(M)$ acting on $M$ on the left. Clearly $M$ is a uniserial right $R$-module.

Fact 2.1. [5, Thm. 9.1] Let $N$ be a uniserial module and let $S=\operatorname{End}(N)$. Then $S$ has at most two maximal right and left ideals: $I$ consisting of nonmonomorphisms, and $K$ consisting of non-epimorphisms.

If $S$ is not local (i.e., if $I$ and $K$ are incomparable), then $S / \mathrm{J}(S)$ is a direct sum of skew fields $S / I$ and $S / K$.

From now on we reserve $R$ for a uniserial domain, $M$ for the module $R / r R, S$ for the endomorphism ring of $M$, and $I, K$ for ideals of $S$ as described in Fact 2.1.

If $f$ is an endomorphism of $M$ such that $f(\overline{1})=\bar{a}, a \in R$, then $f=a \times$ is a left multiplication by $a$, and $a$ is unique modulo $r R$. From $f(\bar{r})=f(\overline{0})=\overline{0}$ it follows that $a r=r a^{\prime}$ for some $a^{\prime} \in R$. Since $R$ is a domain, $a^{\prime}$ is uniquely determined by $a$.

In particular, we may identify $S$ with the 'idealizer' ring $R^{\prime} / r R$, where $R^{\prime}=\{a \in R \mid$ ar $\in r R\}$ and $r R$ is a two-sided ideal of $R^{\prime}$. Then $R^{\prime}=$ $R \cap r R r^{-1}$ is a subring of $D$. 
A ring $T$ is said to be right (left) Bezout, if every finitely generated right (left) ideal of $R$ is principal; and $T$ is Bezout, if it is right and left Bezout. For instance, every (right) uniserial ring is (right) Bezout.

Fact 2.2. [4] $R^{\prime}$ is a Bezout ring with at most two maximal right and left ideals: $I^{\prime}=R \cap r \mathrm{~J}(R) r^{-1}$ and $K^{\prime}=\mathrm{J}(R) \cap r R r^{-1}$.

A ring $T$ is called right (left) $\cap$-Bezout, if the intersection of two principal right (left) ideals of $R$ is a principal right (left) ideal; and $T$ is $\cap$-Bezout, if it is right and left $\cap$-Bezout. Again, every (right) uniserial ring is (right) ก-Bezout.

It is well known (see [3]) that every right Bezout domain is right $\cap$-Bezout. For instance, $R^{\prime}$ is $\cap$-Bezout, hence principal right (and left) ideals of $R^{\prime}$ form a lattice. We conclude that the same is true for $S$.

Corollary 2.3. (cp. [20, L. 5.2]) $S$ is Bezout and $\cap$-Bezout.

Proof. Since $S$ is a factor of $R^{\prime}$ by a principal right ideal $r R$, it readily follows that $S$ is right Bezout and $\cap$-Bezout. The left variant of this property will follow from a general transfer principle (see Lemma 2.6 below).

In the following lemma we identify ideals $I$ and $K$ from Fact 2.1 .

Lemma 2.4. 1) $I=I^{\prime} / r R$, that is, $I$ consists of endomorphisms $f=a \times$ such that ar $=r a^{\prime}$, where $a^{\prime} \in \mathrm{J}(R)$.

2) $K=K^{\prime} / r R$, that is, $K$ consists of endomorphisms $f=a \times$ such that $a \in \mathrm{J}(R)$.

Proof. 1) Suppose that $f=a \times \in I$, i.e., $f$ is not mono. Then $a b=r t$ for some $b \notin r R$ (that is, $\bar{b} \neq 0$ in $M$ and $f(\bar{b})=\overline{a b}=\overline{r t}=0$ ). Since $b \notin r R$, it follows that $r=b g$ for some $g \in \mathrm{J}(R)$. Multiplying $a b=r t$ by $g$ on the right we obtain $a r=r t g$. But also $a r=r a^{\prime}$, hence $a^{\prime}=t g \in \mathrm{J}(R)$.

Now suppose that $a^{\prime} \in \mathrm{J}(R)$. We may assume that $a \times$ is non-zero, that is, $a \notin r R$. Then, by uniseriality, $r=a s$ for some $s \in R$. We prove that $\bar{s} \neq 0$. This would imply $f(\bar{s})=\overline{a s}=\bar{r}=0$, hence $f$ is not mono.

Indeed, otherwise $s=r s^{\prime}$ for some $s^{\prime} \in R$. Then $r=a s=a r s^{\prime}=r a^{\prime} s^{\prime}$. Since $a^{\prime} s^{\prime} \in \mathrm{J}(R), 1-a^{\prime} s^{\prime}$ is a unit. Thus $r\left(1-a^{\prime} s^{\prime}\right)=0$ implies $r=0$, a contradiction.

2) If $a \in \mathrm{J}(R)$, then $\operatorname{im}(f)=\bar{a} R \subseteq \mathrm{J}(R) / r R=\mathrm{J}(M)$ is a proper submodule of $M$.

On the other hand, if $a \notin \mathrm{J}(R)$, then $a$ is a unit. Thus $\overline{1}=f\left(\overline{a^{-1}}\right) \in \operatorname{im}(f)$ yields $\operatorname{im}(f)=M$. 
Let $M^{\prime}$ be the left $R$-module $R / R r$, and let $S^{\prime}=\operatorname{End}\left(M^{\prime}\right)$, where $S^{\prime}$ acts on $M^{\prime}$ on the right. By symmetry, $S^{\prime}$ has a maximal (as a right and as a left) ideal $I^{\prime}$ consisting of non-monomorphisms, and a maximal ideal $K^{\prime}$ consisting of non-epimorphisms.

By arguments symmetric to Lemma 2.4 we obtain the following.

Lemma 2.5. Let $f^{\prime}=\times a^{\prime} \in S^{\prime}$ be such that ar $=r a^{\prime}$. Then $f^{\prime} \in I^{\prime}$ iff $a \in \mathrm{J}(R)$, and $f^{\prime} \in K^{\prime}$ iff $a^{\prime} \in \mathrm{J}(R)$.

The following proposition allows us to transfer left properties of $S$ to the right.

Proposition 2.6. The rule $a \times \mapsto \times a^{\prime}$, where $a r=r a^{\prime}$, defines an isomorphism of rings $\alpha: S \rightarrow S^{\prime}$. This isomorphism alternates monomorphisms and epimorphisms (i.e., identifies $I$ with $K^{\prime}$, and $K$ with $I^{\prime}$ ).

Proof. We check only that $\alpha$ preserves multiplication. Let $f$ be $a \times$, and let $g$ be $b \times$, where $b r=r b^{\prime}$. Then $\alpha(f)$ is $\times a^{\prime}$ and $\alpha(g)$ is $\times b^{\prime}$, hence $\alpha(f) \alpha(g)$ is $\times a^{\prime} b^{\prime}$.

Now $f g$ is $a b \times$. From $a b r=a r b^{\prime}=r a^{\prime} b^{\prime}$ we conclude that $\alpha(f g)$ is $\times a^{\prime} b^{\prime}$, hence $\alpha(f) \alpha(g)=\alpha(f g)$.

By Lemmas 2.4 and 2.5, $\alpha$ alternates monos and epis.

\section{NeARLY SIMPle UNiSERIAL DOMAiN}

Let $R$ be a uniserial domain. Recall that $R$ is nearly simple, if $R$ is not artinian and $\mathrm{J}(R)$ is a unique two-sided ideal of $R$. From now on we reserve $R$ to denote nearly simple uniserial domains. Note that $R$ is never noetherian, moreover (see [19, L. 4.1]) it never has Krull dimension (see [14, Ch. 6] for the definition of Krull dimension): if $a R \subset b R$ are principal right ideals of $R$, then there exists $c \in R$ such that $a R \subset c R \subset b R$, and the same is true for left ideals.

The known examples of nearly simple uniserial domains are constructed using nearly simple cones in groups. We will give one such example following Dubrovin [4]. Note that nearly simple cones form a relatively thin class in the classification of rank one cones in groups [2, p. 2379].

Example 3.1. Let $G$ be the following group of affine linear transformations of $\mathbb{Q}$ considered as a linearly ordered set: $G=\{t \rightarrow a t+b \mid a, b \in \mathbb{Q}, a>0\}$. We use pairs $(a, b)$ to denote elements $a t+b$ of $G$. Therefore $(a, b) \cdot(c, d)=$ $(a c, a d+b),(a, b)^{-1}=\left(a^{-1},-a^{-1} b\right)$ and $(1,0)$ is the unity of $G$. 
Choose an irrational number $\varepsilon$. Then $P=\{g \in G \mid g(\varepsilon) \geq \varepsilon\}$ is a cone in $G$ whose positive cone is $P^{+}=\{g \in G \mid g(\varepsilon)>\varepsilon\}$ (see [2] for the terminology on cones and right ordered groups). If $F$ is a field, then $M=F P^{+}$is a two-sided ideal of the semigroup ring $F P$. Furthermore, $F P \backslash M$ is a (right and left) Ore set such that the corresponding localization $R$ is a nearly simple uniserial domain. For example, from $(t+2)(\varepsilon)>\varepsilon$ it follows that $(1,2) \in P^{+}$, hence $(1,0)+(1,2)$ is a unit of $R$.

Furthermore, every principal right ideal of $R$ is of the form $g R, g \in P^{+}$, and $g R \subseteq h R$ iff $g(\varepsilon) \geq h(\varepsilon)$. Thus the chain of (nontrivial) principal right ideals of $R$ is a dense linear ordering without endpoints, therefore it is isomorphic to $(\mathbb{Q}, \leq)$. The same is true for principal left ideals, but as the following fact shows, right and left principal ideals of $R$ are in a 'generic' position.

Fact 3.2. $[19$, Prop. 6.2] If $0 \neq r, s \in \mathrm{J}(R)$, then $R r+s R=\mathrm{J}(R)$.

The following fact describes the structure of finitely presented modules over a nearly simple uniserial domain.

Fact 3.3. [18, Prop. 14.17] Let $R$ be a nearly simple uniserial domain.

1) If $0 \neq r, s \in \mathrm{J}(R)$, then $R / r R \cong R / s R$.

2) Every finitely presented right $R$-module is a direct sum of (finitely many) copies of $R_{R}$ and $R / r R, 0 \neq r \in \mathrm{J}(R)$, and this decomposition is unique.

In particular, the isomorphism type of $R / r R$ does not depend on a choice of $0 \neq r \in \mathrm{J}(R)$.

A positive primitive formula (pp-formula) $\varphi(x)$ over a $\operatorname{ring} T$ is a formula of the form $\exists \bar{y}(\bar{y} A=x \bar{b})$, where $\bar{y}=\left(y_{1}, \ldots, y_{k}\right)$ is a tuple of bound variables, $A$ is an $k \times l$ matrix over $T$ and $\bar{b}=\left(b_{1}, \ldots, b_{l}\right)$ is a row of elements of $T$. A standard shorthand for this formula is $A \mid x \bar{b}$ ( $A$ divides $x \bar{b})$. For instance, if $a \in T$, then $a \mid x \doteq \exists y(y a=x)$ is a divisibility formula; and if $b \in T$, then $x b=0$ is an annihilator formula.

If $N$ is a $T$-module and $n \in N$, we write $N \models \varphi(n)$ ( $n$ satisfies $\varphi$ in $N)$, if $\bar{n} A=n \bar{b}$ for some tuple $\bar{n}=\left(n_{1}, \ldots, n_{k}\right)$ of elements of $N$. Then $\varphi(N)=$ $\{n \in N \mid N \models \varphi(n)\}$ is a positive-primitive subgroup (pp-subgroup) of $N$, and $\varphi(N)$ is a (left) $\operatorname{End}(N)$-submodule of $N$. For instance, $(a \mid x)(N)=N a$ and $(x b=0)(N)=\operatorname{ann}(N)(b)=\{n \in N \mid n b=0\}$ are pp-subgroups of $N$. 
If $\varphi$ and $\psi$ are pp-formulae, we set $\varphi \rightarrow \psi(\varphi$ implies $\psi)$, if $\varphi(N) \subseteq \psi(N)$ for every module $N$. For instance, if $a, a^{\prime} \in T$, then $a\left|x \rightarrow a^{\prime}\right| x$ iff $a \in T a^{\prime}$; and, if $b, b^{\prime} \in T$, then $x b=0 \rightarrow x b^{\prime}=0$ iff $b^{\prime} \in b T$.

We say that pp-formulae $\varphi$ and $\psi$ are equivalent (written $\varphi \leftrightarrow \psi$ ), if $\varphi \rightarrow \psi$ and $\psi \rightarrow \varphi$, that is, $\varphi(N)=\psi(N)$ for every module $N$. For instance, $a \mid x$ is equivalent to $a^{\prime} \mid x$ iff $T a=T a^{\prime}$, and $x b=0 \leftrightarrow x b^{\prime}=0$ iff $b T=b^{\prime} T$. The set of equivalence classes of all pp-formulae over $T$ ordered by implication forms a modular lattice $L(T)$. The operations in this lattice are as follows: the meet is the conjunction $\varphi \wedge \psi$, and the sum is given by $(\varphi+\psi)(x) \doteq \exists y \varphi(y) \wedge \psi(x-y)$. For instance, $a \mid x+x b=0$ is equivalent to the formula $a b \mid x b$. For more on pp-formulae see [16, Ch. 2].

Having these definitions in mind, one can describe the lattice of ppformulae over a nearly simple uniserial domain.

Fact 3.4. [18, Prop. 14.15] Figure 1 shows the lattice $L$ of all pp-formulae over a nearly simple uniserial domain. The dotted chains are dense linear orderings. Here $a \mid x$ represents the chain of divisibility formulae, which is isomorphic to the chain of left principal ideals of $R$; and $x b=0$ represents the chain of annihilator formulae, which is isomorphic to the chain of right principal ideals of $R$. All the remaining formulae are sums or conjunctions of those.

Let $n$ be an element of a module $N$. The pp-type of $n$ in $N, p p_{N}(n)$, is the set of all pp-formulae $\varphi(x)$ such that $N \models \varphi(n)$. A pp-type $p$ is said to be finitely generated, if there is a pp-formula $\varphi \in p$ such that $\varphi \rightarrow \psi$ for any $\psi \in p$. In this case we say that $\varphi$ generates $p$.

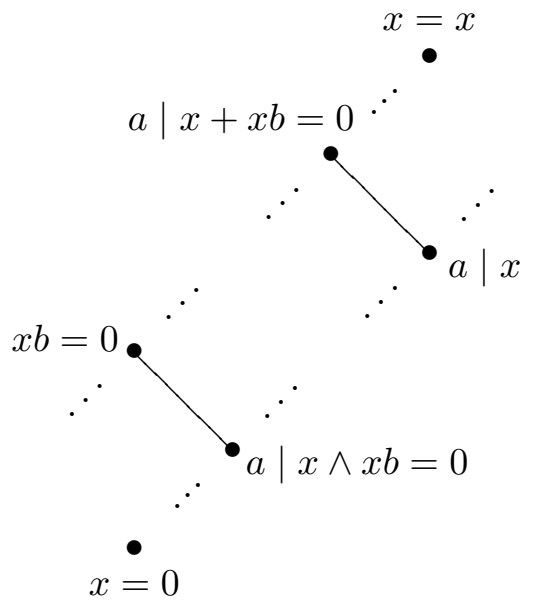

FIGURE 1 
Note that $M=R / r R$ is a finitely (even cyclically) presented module. In particular, by [16, Prop. 8.4], the pp-type of any element of $M$ is finitely generated, hence generated by one pp-formula, which can be read off from Figure 1. For instance, the pp-type of $\overline{1} \in M$ is given by the formula $x r=0$. Furthermore, if $r=a b$ for some $a, b \in R$, then the pp-type of $m=\bar{a} \in M$ is generated by $a \mid x \wedge x b=0$.

Recall that an ideal of a lattice $L$ is a subset of $L$ which is closed with respect to sums and downward directed. For instance, if $a \in L$ then $\{b \in$ $L \mid b \leq a\}$ is an ideal of $L$ generated by $a$. The following remark is crucial in the foregoing calculations.

Remark 3.5. 1) The evaluation map ev $: f \rightarrow f(\overline{1})$ is an isomorphism of left $S$-modules ${ }_{S} S$ and $(x r=0)(M)$.

2) This map induces an isomorphism from the lattice of left principal ideals of $S$ to the ideal of the lattice $L$ generated by $x r=0$.

Proof. 1) For every right $R$-module $N$, the evaluation map provides an isomorphism of left $\operatorname{End}(N)$-modules $\operatorname{Hom}_{R}(M, N)=\operatorname{Hom}(R / r R, N)$ and $(x r=0)(N)$. If $N=M$, then $\operatorname{End}(N)=S$.

2) If $f \in S$, then $f(\overline{1})=m \in(x r=0)(M)$. We set $\operatorname{ev}(S f) \doteq \varphi$, where $\varphi$ generates $p=p p_{M}(m)$, in particular $\varphi \rightarrow x r=0$.

If $g \in S$, then the pp-type of $g f(\overline{1})=g(m)$ in $M$ is generated by a ppformula $\psi$ such that $\psi \rightarrow \varphi$. Thus ev is correctly defined and preserves the ordering.

We check that ev reflects the ordering. Suppose that $\operatorname{ev}(S g)=\psi$ is such that $\psi \rightarrow \varphi$, that is, $g(\overline{1})=n$, where $\psi$ generates $p p_{M}(n)$. Since $M$ is finitely presented, and $\psi \rightarrow \varphi$, by [16, Prop. 8.5], there is $h \in S$ such that $h(m)=n$. Then $h f(\overline{1})=h(m)=n=g(\overline{1})$, hence $h f=g$.

It is easily checked that every pp-formula below $x r=0$ generates the pp-type of some element of $M$, hence ev is onto.

Now we are able to sketch the lattice of left principal ideals of $S$.

Corollary 3.6. [19, Prop. 6.4] Figure 2 represents the lattice of left principal ideals of $S$. Furthermore Figure 3 shows the position of ideals $I$ and $K$ in this lattice. In particular, ${ }_{S} K$ is cyclic and uniserial, and ${ }_{S} I$ is not finitely generated.

Proof. By Remark 3.5, this lattice is isomorphic to the ideal generated by $x r=0$ in the lattice $L$ of all pp-formulae over $R$. Looking at Figure 1, we obtain Figure 2. 


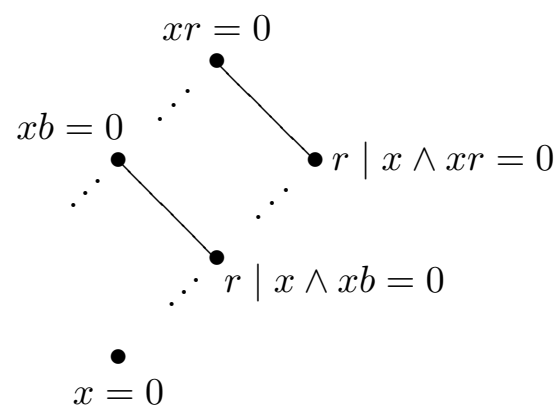

FiguRE 2

Since $M$ is finitely presented, $f \in S g$ for $f, g \in S$ iff $p p_{M}(g(\overline{1})) \subseteq$ $p p_{M}(f(\overline{1}))$. Clearly $f \in K$ iff a nontrivial divisibility formula $a \mid x$ is in $p p_{M}(f(\overline{1}))$. Furthermore, the pp-formulae that imply a non-trivial divisibility formula, form a chain in Figure 2 .

Thus ${ }_{S} K$ is uniserial and generated by any $f \in K \backslash I$.

Similarly, $g \in I$ iff a new annihilator formula (stronger than $x r=0$ ) appears in $p p_{M}(g(\overline{1}))$. Those pp-formulae are not necessarily comparable on Figure 2, hence ${ }_{S} I$ is not uniserial.

Most of results of this paper are valid for any nearly simple uniserial domain $R$. However sometimes we need to put some extra condition on $R$. The most common one will be that $\mathrm{J}(R)$ is countably generated as a right (or left) ideal. Note that this is the case for a nearly simple uniserial domain from Example 3.1. We single out this fact.

Remark 3.7. Let $R$ be a nearly simple uniserial domain from Example 3.1. Then all dotted chains on Figures 1-3 are isomorphic to the ordering of the rationals $(\mathbb{Q}, \leq)$. In particular, every right or left ideal of $R$ and $S$ is countably generated. If the underlying field $F$ is countable, then all these ideals are countable; in particular $R$ and $S$ are countable.
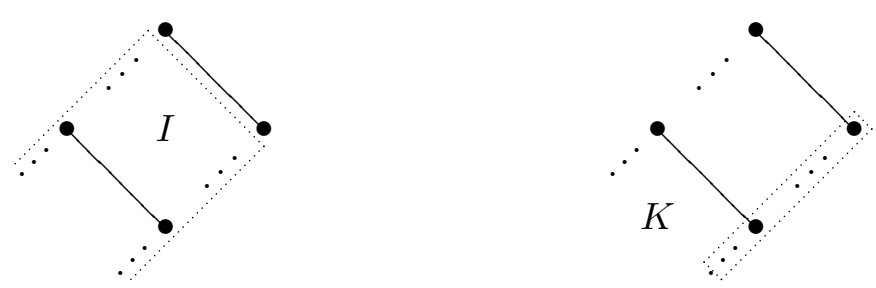

FiguRE 3 
The following example is similar to Example 3.1, but with the lattice of principal one-sided ideals being isomorphic to $(\mathbb{R}, \leq)$.

Example 3.8. Similar to Example 3.1, let $G=\{a t+b \mid a, b \in \mathbb{R}, a>0\}$ be a group of affine linear transformations of $(\mathbb{R}, \leq)$. Then $P=\{g \in G \mid$ $g(\varepsilon) \geq \varepsilon\}$ defines a cone on $G$, that is, $P \cdot P \subseteq P$ and $P \cup P^{-1}=G$. If $F$ is a field, then, as in the same example, we can localize the semigroup ring FP by a maximal two-sided ideal to obtain a nearly simple uniserial domain $R$; and every principal right ideal of $R$ is generated by some $g \in P$.

However it is more complicated to decide the inclusion of such ideals in this case. Namely, the cone $P$ is not pure, that is, $H=P \cap P^{-1}=\{g \in$ $G \mid g(\varepsilon)=\varepsilon\}$ is a nontrivial (non-normal) subgroup of $G$, therefore right principal ideals of $R$ correspond to right cosets of $H$ in $G$. Indeed, for every $(a, b) \in G$ there exists a unique $(a, c) \in H \subseteq \mathrm{U}(R)$. Then $(a, b) R=$ $(a, b)(a, c)^{-1} R=(1, d) R$ for some $d \in \mathbb{R}$, and $d_{1} \leq d_{2}$ iff $\left(1, d_{1}\right) R \supseteq\left(1, d_{2}\right) R$. Thus the chain of (nontrivial) principal right ideals of $R$ is isomorphic to $(\mathbb{R}, \leq)$.

\section{Some PRELIMINARIES}

If $f \notin K$ (that is, if $f$ is an epimorphism), then the left annihilator of $f$, $\operatorname{ann}(S)(f)$, is zero. Otherwise $f$ is non-epi, and we calculate the left annihilator of $f$ in the following lemma.

Lemma 4.1. If $0 \neq f \in K$, then $\operatorname{ann}(S)(f)=S g$ for some $g \in I \backslash K$ such that $\operatorname{im}(f)=\operatorname{ker}(g)$.

Proof. Since $\operatorname{im}(f)$ is a cyclic proper submodule of $M$, it follows that $M / f(M)$ is a nonzero finitely presented uniserial module. Since $M / f(M)$ is torsion, Fact 3.3 yields $M / \operatorname{im}(f) \cong M$.

Combining the projection $M \rightarrow M / \operatorname{im}(f)$ with an isomorphism $M / \operatorname{im}(f) \rightarrow$ $M$, we obtain an epimorphism $g: M \rightarrow M$ such that $\operatorname{ker}(g)=\operatorname{im}(f)$. Clearly $g$ is the cokernel of $f$ in the category of all $R$-modules. Then every map $h: M \rightarrow M$ such that $h f=0$ can be factored through $g$.

If $g \notin I$ (that is, if $g$ is a monomorphism), then the right annihilator of $g$, $\operatorname{ann}(g)(S)$, is zero.

The following lemma is dual to Lemma 4.1.

Lemma 4.2. If $0 \neq g \in I$, then $\operatorname{ann}(g)(S)=f S$ for some $f \in K \backslash I$ such that $\operatorname{ker}(g)=\operatorname{im}(f)$. 
Now we give an example of calculations of $f$ and $g$.

Example 4.3. Let $R$ be a nearly simple uniserial domain from Example 3.1, and choose $\varepsilon=1 / \sqrt{2}, r=(1,1)$. Since $r(\varepsilon)=(t+1)(\varepsilon)>\varepsilon$, therefore $0 \neq r \in \mathrm{J}(R)$ and we set $M=R / r R$.

In what follows we calculate in $D$, the quotient (skew) field of $R$. As we have already noticed, if $d \in D \backslash R$, then $d^{-1} \in \mathrm{J}(R)$. From $(5 t-4)(\varepsilon)<\varepsilon$ it follows that $(1,0)+(5,-4) \notin R$, hence $s=((1,0)+(5,-4))^{-1} \in \mathrm{J}(R)$. Another way to see this is to notice that $s=[(1,0)+(1 / 4,4 / 5)]^{-1}(1 / 5,4 / 5)=$ $(1 / 5,4 / 5)[(1,0)+(1 / 5,4 / 5)]^{-1}$.

Let $f$ be given by left multiplication by $s$. We claim that $f$ defines a monomorphism of $M$ which is not epi. Since $s \in \mathrm{J}(R)$, by Lemma 2.4 and Fact 2.2, it suffices to check that $s \in r R r^{-1}$, that is $v=r^{-1} s r \in R$. But $v^{-1}=r^{-1} s^{-1} r=(1,-1)[(1,0)+(5,-4)](1,1)=(1,0)+(5,0) \in \mathrm{U}(R)$, hence $v \in \mathrm{U}(R)$.

Similarly, since $(t / 5+3 / 5)(\varepsilon)>\varepsilon$, we conclude that $(1 / 5,3 / 5) \in \mathrm{J}(R)$, hence $u=[(1,0)+(1 / 5,3 / 5)]^{-1} \in \mathrm{U}(R)$. Let $g$ be given by left multiplication by $u$. We prove that $g$ defines a monomorphism of $M$ which is not mono. By Lemma 2.4 and Fact 2.2 again, it suffices to show that $u \in r \mathrm{~J}(R) r^{-1}$, that is, $r^{-1} u r \in \mathrm{J}(R)$ or $r^{-1} u^{-1} r \notin R$. Indeed, $r^{-1} u^{-1} r=(1,-1)[(1,0)+$ $(1 / 5,3 / 5)](1,1)=(1,0)+(1 / 5,-1 / 5) \notin R$, since $(t / 5-1 / 5)(\varepsilon)<\varepsilon$.

It remains to check that $\operatorname{im}(f)=\operatorname{ker}(g)$. Clearly the kernel of $f$ is generated by $u^{-1} r=[(1,0)+(1 / 5,3 / 5)](1,1)=(1,1)+(1 / 5,4 / 5)$, hence $\operatorname{ker}(g)=(1 / 5,4 / 5) R+r R$.

On the other hand, since $s=(1 / 5,4 / 5)[(1,0)+(1 / 5,4 / 5)]^{-1}$, the image of $f$ is equal to $s R+r R=(1 / 5,4 / 5)+r R$, hence $\operatorname{im}(f)=\operatorname{ker}(g)$, as desired.

There is a long list of known properties of $S$. For instance, by [20, Cor. 6.5], $S$ is left and right uniform, $I$ is the right singular ideal of $S$, and $K$ is the left singular ideal of $S$. We add one more property to this list.

Lemma 4.4. $S$ is left and right coherent.

Proof. By Chase's criterion (see [12, Cor. 4.60]) a ring $S$ is right coherent iff 1) the intersection of two finitely generated right ideals of $S$ is a finitely generated right ideal, and 2) the right annihilator $\operatorname{ann}(g)(S)$ of any $g \in S$ is a finitely generated right ideal.

By Corollary 2.3, $S$ is right Bezout and right $\cap$-Bezout, hence 1) holds true. Furthermore, 2) follows from Lemma 4.2. 
Thus $S$ is right coherent. By symmetric arguments $S$ is left coherent, hence coherent.

The following remark shows that the kernel of an endomorphism $f$ of $M$ can be recognized by the annihilator of $m=f(\overline{1})$.

Remark 4.5. Let $f \in S$ and let $m=f(\overline{1})$. Then $\bar{a} \in \operatorname{ker}(f)$ if and only if $m a=0$.

Proof. If $\bar{a} \in \operatorname{ker}(f)$, then $0=f(\bar{a})=f(\overline{1}) a=m a$. The converse is also clear.

If $I$ is a right ideal of $S$, the double annihilator of $I$ is a right ideal $\operatorname{ann}(J)(S)$, where $J=\operatorname{ann}(S)(I)$ is the left annihilator of $I$. For instance, if $f \notin K$ (i.e., if $f$ is epi) and $I=f S$, then $J=0$, hence the double annihilator of $I$ is $S$. The double annihilator of a left ideal of $S$ is defined similarly.

Corollary 4.6. 1) If $f \in K \backslash I$ (i.e., if $f$ is non-epi and mono), then the double annihilator of $f S$ is equal to $f S$. In particular, $f S=\operatorname{ann}(g)(S)$ for some $g \in K \backslash I$ such that $\operatorname{im}(f)=\operatorname{ker}(g)$.

2) If $g \in I \backslash K$ (i.e., if $g$ is non-mono and epi), then the double annihilator of $S g$ is equal to $S g$. In particular, $S g=\operatorname{ann}(S)(f)$ for some $f \in K \backslash I$ such that $\operatorname{ker}(g)=\operatorname{im}(f)$.

Proof. We prove only 2). The arguments for 1) are similar.

By Lemma 4.2, $\operatorname{ann}(g)(S)=f S$ for some $f \in K \backslash I$ such that $\operatorname{ker}(g)=$ $\operatorname{im}(f)$. Furthermore, by Lemma 4.1, $\operatorname{ann}(S)(f)=S g^{\prime}$ for some $g^{\prime} \in I \backslash K$ such that $\operatorname{im}(f)=\operatorname{ker}\left(g^{\prime}\right)$. Then $\operatorname{ker}(g)=\operatorname{ker}\left(g^{\prime}\right)$.

By Remark 4.5, the pp-types of $g(\overline{1})$ and $g^{\prime}(\overline{1})$ in $M$ contain the same annihilator formulae. Since $g, g^{\prime} \notin K$, these pp-types contain no non-trivial divisibility formula. Looking at the lattice of pp-formulae over $R$ (Figure 1), we conclude that $g(\overline{1})$ and $g^{\prime}(\overline{1})$ have the same pp-type. Since $M$ is finitely presented, it follows readily that $S g=S g^{\prime}$.

\section{Finitely PResented modules}

In this section we describe finitely presented $S$-modules. As a first approximation we prove the following.

Proposition 5.1. Every square matrix over $S$ can be diagonalized by elementary row and column operations. 
Proof. Suppose that $A$ is an $n \times n$ matrix over $S$, and we apply induction on $n$.

Step 1. Suppose that some entry $a_{i j}$ of $A$ is a unit.

Then, by elementary operations of rows and columns, we can kill all the elements, but $a_{i j}$, in the $i$ th row and the $j$ th column. After that apply induction to the minor $A_{i j}$.

Step 2. Suppose that the $i$ th row of $A$ contains an element $a_{i j} \in K \backslash I$, and also contains an element $a_{i k} \in I \backslash K$.

Then, adding the $j$ th column to the $k$ th, we obtain the unit $a_{i j}+a_{i k}$ on the $i k$ position. Thus we can reduce the size of the matrix as in Step 1.

Similarly, we may reduce the size of $A$, if some column of $A$ contains an element from $K \backslash I$ and an element from $I \backslash K$.

Step 3. Suppose that $A$ contains elements from $K \backslash I$ and from $I \backslash K$, say, $a_{i j} \in K \backslash I$ and $a_{k l} \in I \backslash K$. If $i=k$ (that is, these elements are in the same row), or $j=l$ (that is, these elements are in the same column), we reduce the size of the matrix as in Step 2.

Thus, we may assume that $i \neq k$ and $j \neq l$. Consider $a_{i l}$. If $a_{i l}$ is invertible, we proceed as in Step 1.

If $a_{i l} \in K \backslash I$ then, since $a_{k l} \in I \backslash K$, we follow Step 2 again. Similarly, if $a_{i l} \in K \backslash I$, we can use $a_{i j} \in I \backslash K$ to obtain a unit in the $i$ th row of $A$.

$$
\begin{array}{ccc}
a_{i j} & & a_{i l} \\
\vdots & & \vdots \\
a_{k j} & \ldots . & a_{k l}
\end{array}
$$

Thus we may assume that $a_{i l} \in \mathrm{J}(S)$. By similar arguments we may suppose that $a_{k j} \in \mathrm{J}(S)$. Then, adding the $k$ th row to the $i$ th, we obtain $a_{i j}+a_{k j} \in K \backslash I$ as the $i j$-entry; and $a_{i l}+a_{k l} \in I \backslash K$ as the $i$-entry. Thus we may proceed as in Step 2 again.

Step 4. Therefore we may assume that either all the entries of $A$ are in $K$, or all the entries of $A$ are in $I$. Because the arguments below are symmetric, let us assume the former.

If all the entries of $A$ are in $\mathrm{J}(S)$, then, since $\mathrm{J}(S)$ is a uniserial left and right $S$-module, the proof of [18, Thm. 2.1] shows that $A$ is diagonalizable by elementary operations of rows and columns.

Otherwise we may assume that $a_{1}=a_{11} \in K \backslash I$. Then $S a_{1}=K$, hence, using the first row, we can kill all the elements, but $a_{1}$, in the first column.

Applying induction to the minor $A_{11}$, we may further suppose that $A$ has the following form: 


$$
\left(\begin{array}{cccc}
a_{1} & a_{2} & \ldots & a_{n} \\
0 & b_{2} & \ldots & 0 \\
\vdots & \vdots & \ddots & \vdots \\
0 & 0 & \ldots & b_{n}
\end{array}\right)
$$

Adding, if necessary, first column, we may assume that $a_{i} \in K \backslash I$ for every $i$. Then the right ideals $a_{i} S$ are linearly ordered by inclusion (since the left ideals $S g, g \in I \backslash K$ are linearly ordered by inclusion, and apply the isomorphism $S \rightarrow S^{\prime}$ ).

If $a_{i} \in S b_{i}$ for some $i \geq 2$, we can kill $a_{i}$ using the $i$ th row, and then apply induction to the minor $A_{i i}$. Therefore, since ${ }_{S} K$ is uniserial, we may assume that $b_{i}=c_{i} a_{i}, c_{i} \in S$ for every $i \geq 2$.

Now, if $a_{i} \in a_{1} S$ for some $i \geq 2$, we can kill this $a_{i}$ using the first column, and apply induction to the minor $A_{i i}$. Otherwise we may assume that $a_{i} \in a_{2} S$ for every $i$. Then (since $b_{2}=c_{2} a_{2}$ ) we can kill $b_{2}$, using the first row, and after that kill all the $a_{i}, i \neq 2$, using the second column.

It remains to apply induction to the minor $A_{12}$.

The following is a standard conclusion.

Corollary 5.2. Every finitely presented left $S$-module is isomorphic to a finite direct sum of modules $S / S s_{i}, s_{i} \in S$.

Recall that $S$ has two simple modules: $S / K$ and $S / I$. The following lemma shows that one of them is injective.

Lemma 5.3. $S / K$ is a simple injective left $S$-module, and $S / I$ is a simple injective right $S$-module.

Proof. $S / K$ is a simple module over its endomorphism ring $S / K$. It follows easily that $S / K$ is pure-injective. By [11, Prop. 7.23], a pure-injective module is injective iff it is injective with respect to finitely generated ideals. Since $S$ is Bezout, it suffices to check that $S / K$ is injective with respect to principal ideals of $S$.

Thus, let $h \in S, \bar{s} \in S / K$ be such that $\operatorname{ann}(S)(h) \subseteq \operatorname{ann}(S)(\bar{s})$ (i.e., $\bar{s}$ is divided by $h$ in some extension of $S / K)$, and we have to prove that $\bar{s} \in h(S / K)$. We may assume that $\bar{s} \neq 0$, hence $\operatorname{ann}(S)(\bar{s})=K$.

From Lemma 4.1 it follows that $h \notin K$. Since $S / K$ is a simple right $S$-module, there is $t \in S$ such that $\bar{h} t=\bar{s}$. Then $h \bar{t}=\bar{s}$, which shows divisibility.

$S / I_{S}$ is injective by symmetric arguments. 
Lemma 5.4. If $h \in \mathrm{J}(S)$, then $0 \neq h=f g^{\prime}$ for some $f \in K \backslash I$, and $g^{\prime} \in I \backslash K$.

Proof. As in Corollary 4.6 we have $\operatorname{ann}(h)(S)=f^{\prime} S$ for some $f^{\prime} \in K \backslash I$, and $\operatorname{ann}(S)\left(f^{\prime}\right)=S g^{\prime}$, where $g^{\prime} \in I \backslash K$ is such that $\operatorname{ker}(h)=\operatorname{ker}\left(g^{\prime}\right)$.

Let $p=p p_{M}(h(\overline{1}))$ and $q=p p_{M}\left(g^{\prime}(\overline{1})\right)$. These pp-types have the same annihilator formulae. From $h \in \mathrm{J}(S)$ it follows that $p$ contains a nontrivial divisibility formula, but $q$ is not. Looking at the lattice of pp-formulae (Figure 1), we see that $q \subset p$.

Then there exists $f \in S$ which sends $g^{\prime}(\overline{1})$ to $h(\overline{1})$, therefore $f g^{\prime}=h$. Since $f$ adds a new divisibility formulae to $q$, we conclude that $f \in K$. Furthermore, $f$ does not change the annihilator part of $p$, hence $f \notin I$.

Now we are in a position to describe the structure of finitely presented left $S$-modules.

Theorem 5.5. Every finitely presented left $S$-module is isomorphic to a finite direct sum of modules ${ }_{S} S,{ }_{S} S / K$, and ${ }_{S} K$. Furthermore, this decomposition is unique.

Proof. By Corollary 5.2, every finitely presented left $S$-module is a finite direct sum of modules $S / S s, s \in S$.

If $s=0$, then $S / S s=S$, and, if $s$ is invertible, then $S / S s$ is a zero module. Thus we may assume that $s \neq 0$, and $s$ is not a unit.

If $s=f \in K \backslash I$, then $S f=K$, hence $S / S s=S / K$ is a simple left $S$-module.

Suppose that $s=g \in I \backslash K$. Then, by Lemma 4.6, $S g=\operatorname{ann}(S)(f)$ for some $f \in K \backslash I$, hence $S / S g \cong S f=K$.

Otherwise $s=h \in \mathrm{J}(S)$. We prove that $S / S h \cong S / K \oplus K$.

By Lemma $5.4, h=f g^{\prime}$, where $f \in K \backslash I$ and $g^{\prime} \in I \backslash K$. Then $S g^{\prime} / S h=$ $S g^{\prime} / S f g^{\prime} \cong S / S f=S / K$ is a simple injective submodule (see Lemma 5.3), hence a direct summand of $S / S h$. The complement of $S / K$ in $S / S h$ is isomorphic to $S / S g^{\prime} \cong K$.

It remains to prove the uniqueness of this decomposition. Suppose that $S^{m} \oplus(S / K)^{n} \oplus K^{k}$ is isomorphic to $S^{m^{\prime}} \oplus(S / K)^{n^{\prime}} \oplus K^{k^{\prime}}$. Since $S / K$ is simple, it has the exchange property (for the definition of the exchange property see $\left[5\right.$, p. 33]). It readily follows that $n=n^{\prime}$. After cancelation, we may assume that $n=n^{\prime}=0$.

Since $S$ is a semilocal ring, by [5, Cor. 4.6], ${ }_{S} S$ has a cancelation property, and the same is true for a uniserial module ${ }_{S} K$. Furthermore, ${ }_{S} S$ is a 
uniform module of dual Goldie dimension 2. This clearly leads to $m=m^{\prime}$ and $k=k^{\prime}$.

By symmetry, we obtain the following.

Corollary 5.6. Every finitely presented right $S$-module is isomorphic to a finite direct sum of modules $S_{S}, S / I_{S}$, and $I_{S}$, and this decomposition is unique.

\section{Projective dimension}

In this section we calculate the projective dimension of $S$. We choose $f \in K \backslash I$, in particular, $S f=K$ and $S / K=S / S f$. By Lemma 4.1, $\operatorname{ann}(S)(f)=S g$ for some $g \in I \backslash K$ such that $\operatorname{im}(f)=\operatorname{ker}(g)$. Then $K=S f \cong S / S g$. In all statements of this section we keep this notation.

Lemma 6.1. 1) The flat and projective dimension of ${ }_{S} K$ is equal to 1.

2) The flat and projective dimension of ${ }_{S} S / K$ is equal to 2.

Proof. 1) Since $g$ is left non-zero divisor, $S g \cong S$ is a free module. Then the short exact sequence $0 \rightarrow S g \rightarrow S \rightarrow S f=K \rightarrow 0$ shows that both flat and projective dimension of ${ }_{S} K$ does not exceed 1. It remains to check that $K$ is not flat as a left $S$-module. Indeed, otherwise the above short exact sequence would be pure, hence split (since every pure embedding of finitely presented modules splits). But $S$ has no nontrivial idempotents, a contradiction.

2) follows from 1) by standard shift arguments.

Now we are in a position to calculate the weak and global dimensions of $S$. By Jensen (see [10, Thm. 1]), if all the ideals of a ring $T$ are countably generated, then the weak and the global dimension of $T$ could not differ by more than one. For our ring $S$ they coincide.

Proposition 6.2. 1) $S$ has weak dimension 2.

2) If ${ }_{R} \mathrm{~J}(R)$ is countably generated then the left global dimension of $S$ is also 2 .

Proof. 1) By [13, Thm. 5.63], the weak dimension of a ring is left-right symmetric. Furthermore, by [13, Thm. 5.64], it suffices to check that the flat dimension of every left ideal $M$ of $S$ does not exceed 1 . If $M=K$, it follows from Lemma 6.1. Suppose that $M \neq K$ is not contained in $\mathrm{J}(S)$. Then (see Figure 3) $M$ is a union of ideals of the form $S g^{\prime}, g^{\prime} \in I \backslash K$. But 
every such ideal is isomorphic to $S$, hence free. Thus $M$ is flat being a union of a directed system of free modules.

It remains to consider the case when $M \subseteq \mathrm{J}(S)$. Looking at Figure 3 again, we see that $M=N \cap K$, where $N$ is a left ideal of $S$ containing some element from $I \backslash K$, hence $N+K=S$. By what we have already proved, $N$ is flat, and $K$ has flat (and projective) dimension 1 by Lemma 6.1 . Since the following short exact sequence $0 \rightarrow M=N \cap K \rightarrow N \oplus K \rightarrow N+K=S \rightarrow 0$ splits, it follows that the flat dimension of $M$ is at most 1 , as desired.

2) Arguing as in the proof of 1 ) we may assume that $M \neq K$ is not contained in $\mathrm{J}(S)$, and we have to prove that $M$ has projective dimension one. Looking at Figure 3 we conclude that $M$ has a countable system of generators $y_{1}, y_{2}, \ldots \in I \backslash K$ and relations $y_{1}=g_{2} y_{2}, y_{2}=g_{3} y_{3}, \ldots$, where $g_{i} \in I \backslash K$. Map a free module $S^{(\omega)}$ onto $M$ by sending $x_{i}$ (the canonical generator of $\left.S^{(\omega)}\right)$ to $y_{i}$. The kernel of this map is generated by the following elements: $x_{1}-g_{2} x_{2}, x_{2}-g_{3} x_{3}, \ldots$ It follows easily that this kernel is free, hence $M$ has projective dimension (at most) 1 , as desired.

We have already seen that $K$ is not flat as a left $S$-module. To prove that $K_{S}$ is a projective module, we need an auxiliary lemma.

Lemma 6.3. Let $m \in \mathrm{J}(M)$. Then there exists $f \in K \backslash I$ such that $f(m)=$ $m$.

Proof. Let $m=\bar{a}$, where $a \in \mathrm{J}(R)$. We may assume that $m \neq 0$, i.e. $a \notin r R$. Then $a g=r$ for some $g \in \mathrm{J}(R)$. Take any $0 \neq w \in \mathrm{J}(R)$. By Fact 3.2, $\mathrm{J}(R)=R w a+r R$, hence $s w a=a+r t$ for some $s, t \in R$. Multiplying this by $g$ on the right, we obtain $s w r=r(1+t g)$.

Thus, left multiplication by $s w$ defines an endomorphism $f \in S$. Since $s w \in \mathrm{J}(R), f$ is not epi. Since $1+t g$ is a unit, by Lemma $2.4, f$ is mono. Now $f(m)=f(\bar{a})=\overline{s w a}=\overline{a+r t}=\bar{a}=m$.

Now we prove the promised result.

Lemma 6.4. If $\mathrm{J}(M)_{R}$ is countably generated, then $K$ is projective as a right $S$-module.

Proof. (cf. [7, Thm. 5.3]) Let $m_{1}, m_{2}, \ldots$ be a sequence of generators for $\mathrm{J}(M)$. Choose $f_{1} \in K \backslash I$ such that $f_{1}\left(m_{1}\right)=m_{1}$ (see Lemma 6.3). Since $\operatorname{im}\left(f_{1}\right) \subset \mathrm{J}(M)$, we may assume that $m_{2} \notin \operatorname{im}\left(f_{1}\right)$. Take $f_{2} \in K \backslash I$ such that $f_{2}\left(m_{2}\right)=m_{2}$, and so on. 
Clearly $f_{k+1} f_{k}=f_{k}$ for every $k$. We prove that the $f_{i}$ generate $K$. Indeed, if $f \in K$, then $\operatorname{im}(f) \subseteq m_{k} R$ for some $k$, hence $f=f_{k} f \in f_{k} S$.

Now $K$ is projective using standard arguments (see [7, L. 3.1]).

For instance, the proof of this lemma shows that $K$ is an idempotent ideal.

\section{Projective modules}

First we recall the following important fact.

Fact 7.1. [17] If $P$ and $Q$ are projective modules over an arbitrary ring, then $P / \mathrm{J}(P) \cong Q / \mathrm{J}(Q)$ implies $P \cong Q$.

For semilocal rings this fact has a very simple reformulation. Suppose $T$ is a semilocal ring with simples $S_{1}, \ldots, S_{n}$. If $P$ is a projective $T$-module, then the factor $P / \mathrm{J}(P)=P / P \mathrm{~J}(T)$ is a direct sum of the $S_{i}: P / \mathrm{J}(P)=$ $S_{1}^{\left(\alpha_{1}\right)} \oplus \cdots \oplus S_{n}^{\left(\alpha_{n}\right)}$, where $\alpha_{i}$ are cardinals. We call the vector $\left(\alpha_{1}, \ldots, \alpha_{n}\right)$ the dimension vector of $P, \operatorname{dim}(P)$. For instance, if $P$ is a finitely generated projective module, then each coordinate in the dimension vector of $P$ is finite; and each coordinate in the dimension vector of $T$ is nonzero.

Over a semilocal ring projective modules are determined by their dimension vectors.

Corollary 7.2. If $P$ and $Q$ are projective modules over a semilocal ring, then $P \cong Q$ if and only if $\operatorname{dim}(P)=\operatorname{dim}(Q)$.

If $T$ is a semilocal ring, then the finite projective spectrum of $T$ is the collection of the dimension vectors of all finitely generated projective $T$ modules. For purpose of this paper only we will define the infinite projective spectrum of $T$ as the set of all dimension vectors of non-finitely generated projective $T$-modules without finitely generated direct summands. Finally, the spectrum of $T$ is the collection of the dimension vectors of all projective $T$-modules.

In this short section we recall a classification of projective $S$-modules, hence give an example of the spectrum of a semilocal ring. The result has been already known from [19] (see also [17]), and the arguments are similar to those applied by Př́hoda. But we consider them as a warm-up before the next section. To avoid technicalities, we assume that $\mathrm{J}(R)$ is countably generated on the right. Note that this holds for the nearly simple uniserial domain from Example 3.1. 
Theorem 7.3. Every projective right $S$-module $P$ is isomorphic to the module $S_{S}^{(\alpha)} \oplus K_{S}^{(\beta)}$.

Proof. Since $S$ has only two maximal ideals, the dimension vector of every projective $S$-module $P$ has only two coordinates: $\operatorname{dim}(P)=(\alpha, \beta)$. Furthermore, since $S / I$ and $S / K$ are fields, $\operatorname{dim}(S)=(1,1)$.

By Kaplansky's theorem (see [5, Cor. 2.48]), $P$ is a direct sum of countably generated modules. Thus we may assume that $P$ is countably generated, hence $\alpha(P), \beta(P) \leq \omega$.

If $\operatorname{dim}(P)=(\omega, \omega)=\operatorname{dim}\left(S^{(\omega)}\right)$, then $P$ is free by Corollary 7.2. It is also known (see $[20$, L. 2.6]), that $\alpha(P) \geq \beta(P)$. Thus we may assume that $\beta(P)=k$ is finite.

'Subtracting' $k$ copies of $S$ (that is, using projectives covers), it is easily shown that $P \cong S^{k} \oplus P^{\prime}$, where $\beta\left(P^{\prime}\right)=0$.

On the other hand, by [20, Ex. 6.2] there exists a (countably generated) projective $S$-module $V$ with dimension vector $(1,0)$. If $\alpha=\alpha\left(P^{\prime}\right)$, then $P^{\prime} \cong V^{(\alpha)}$ by Corollary 7.2 again.

Corollary 7.4. The projective spectrum of $S$ is a cone $\{(\alpha, \beta) \mid \alpha \geq \beta\}$, where $\alpha$ and $\beta$ are arbitrary cardinals. Furthermore, the finite projective spectrum of $R$ consists of vectors $(n, n), n<\omega$; and the infinite projective spectrum of $S$ consists of vectors $(\delta, 0)$.

Proof. Indeed, $\operatorname{dim}(S)=(1,1)$ and $\operatorname{dim}(V)=(1,0)$, hence $\operatorname{dim}\left(S^{(\alpha)} \oplus V^{\beta}\right)=$ $(\alpha+\beta, \beta)$. Furthermore, as follows from the proof of Theorem 7.3 (or see [20, L. 2.6]), every finitely generated projective $S$-module $P$ is free, hence $\operatorname{dim}(P)=(n, n)$ for some $n<\omega$.

\section{Pure-projective $S$-modules. Dimension.}

A module $N$ is called pure-projective, if $N$ is projective with respect to pure short exact sequences. By Warfield (see [5, Prop. 1.24]) $N$ is pureprojective iff $N$ is a direct summand of a direct sum of finitely presented modules. In particular, every finitely presented module is pure-projective.

If $N$ is a right $T$-module, $\operatorname{add}(N)$ will denote the full subcategory of the category of right $T$-modules consisting of direct summands of finite direct sums of copies of $N$. Similarly, if we allow to take arbitrary direct sums in this definition, we obtain the category $\operatorname{Add}(N)$.

Remark 8.1. A right $S$-module $N$ is pure-projective if and only if $N$ is in $\operatorname{Add}\left(S_{S} \oplus I_{S} \oplus S / I_{S}\right)$. 
Proof. By Corollary 5.6, every finitely presented right $S$-module is a finite direct sum of modules $S_{S}, I_{S}$, and $S / I_{S}$; and apply Warfield's result.

The objective of the next two sections is to give a complete classification of pure-projective right $S$-modules.

We begin with the following general lemma.

Lemma 8.2. Let $N, L$ be right modules over a ring $T$ such that $\operatorname{Hom}(L, N)=$ 0 . Then $\operatorname{add}(N \oplus L)=\operatorname{add}(N) \oplus \operatorname{add}(L)$. Furthermore, if $N$ and $L$ are finitely generated, then $\operatorname{Add}(N \oplus L)=\operatorname{Add}(N) \oplus \operatorname{Add}(L)$.

Proof. Let $U=\operatorname{End}(N \oplus L)$. Then (see [5, Thm. 4.7]), the functor $\operatorname{Hom}(N \oplus$ $L,-)$ gives an equivalence between $\operatorname{add}(N \oplus L)$, and the category of finitely generated projective right $U$-modules.

If $N$ and $L$ are finitely generated, this gives raise to an equivalence between $\operatorname{Add}(N \oplus L)$, and the category of all projective right $U$-modules. We give the proof only in this case.

Since $\operatorname{Hom}(L, N)=0$, we conclude that $U=\left(\begin{array}{cc}\operatorname{End}(N) & 0 \\ \operatorname{Hom}(N, L) & \operatorname{End}(L)\end{array}\right)$, acting on the column $\left(\begin{array}{l}N \\ L\end{array}\right)$ by multiplication on the left. If $e_{1}=e_{11}$ and $e_{2}=e_{22}$ are matrix units, then $U_{12}=e_{1} U e_{2}=0$.

Note that $N$ goes to $\operatorname{Hom}(N \oplus L, N)$, that is, to $e_{1} U$ under this equivalence, and $L$ goes to $e_{2} U$. Thus, it suffices to prove that every projective right $U$-module is a direct sum of modules in $\operatorname{Add}\left(e_{1} U\right)$ and $\operatorname{Add}\left(e_{2} U\right)$.

Since $U_{12}=0$, it follows that $X=e_{2} U$ is a two-sided ideal in $U$ such that $U / e_{2} U=e_{1} U=e_{1} U e_{1}=U_{1}$ is a ring. Thus every $U_{1}$-module is a $U$-module (with respect to the projection $U \rightarrow U_{1}$ ).

Since $U_{1}$ is a projective right $U$-module isomorphic to $e_{1} U$, every projective right $U_{1}$-module is a projective right $U$-module in $\operatorname{Add}\left(e_{1} U\right)$.

Let $P$ be a projective right $U$-module. Then $P / P X$ is a projective $U / X=$ $U_{1}$-module, hence $P / P X$ is a projective right $U$-module in $\operatorname{Add}\left(e_{1} U\right)$. Then the short exact sequence $0 \rightarrow P X \rightarrow P \rightarrow P / P X \rightarrow 0$ splits: $P=P X \oplus Q$, where $Q \cong P / P X \in \operatorname{Add}\left(e_{1} U\right)$, hence $Q X=0$. Multiplying this decomposition by $X$ on the right, we obtain $P X=P X^{2}$, i.e. $P^{\prime}=P^{\prime} X$, where $P^{\prime}=P X$.

It remains to prove that $P^{\prime} \in \operatorname{Add}\left(e_{2} U\right)$. Since $P^{\prime}$ is projective, there are morphisms $\alpha: P^{\prime} \rightarrow U^{(I)}$ and $\beta: U^{(I)} \rightarrow P^{\prime}$ such that $\beta \alpha=1_{P^{\prime}}$. Since $P^{\prime}=P^{\prime} X$, every $m \in P^{\prime}$ can be written as $m=\sum_{i} m_{i} x_{i}$, where $m_{i} \in P^{\prime}$ and $x_{i} \in X$. Then $\alpha(m)=\sum_{i} \alpha\left(m_{i}\right) x_{i} \in U^{(I)} X \subseteq e_{2} U^{(I)}$ (since $X=e_{2} U$ 
is a two-sided ideal). Therefore $\alpha\left(P^{\prime}\right) \subseteq e_{2} U^{(I)}$, hence the restriction of $\beta$ on $e_{2} U^{(I)}$ splits $\alpha$.

Question 8.3. Is Lemma 8.2 true without any restriction on $N$ and $L$ ?

Recall that $S / I$ is a simple injective right $S$-module (by Lemma 5.3). First we get rid of the injective component.

Corollary 8.4. Every pure-projective right $S$-module is a direct sum of a (pure-projective) module in $\operatorname{Add}(S \oplus I)$ and the copies of $S / I$.

Proof. By Remark 8.1, every pure-projective right $S$-module is in $\operatorname{Add}(S \oplus$ $I \oplus S / I)$.

Recall that $g$ is (any) element from $I \backslash K$. Since $g$ is a left non-zero divisor, and $I=g S$, it follows that $\operatorname{Hom}(S / I, S \oplus I)=0$. By Lemma 8.2, we obtain $\operatorname{Add}(S \oplus I \oplus S / I)=\operatorname{Add}(S \oplus I) \oplus \operatorname{Add}(S / I)$. But every module in $\operatorname{Add}(S / I)$ is a vector space over the skew field $S / I$.

Thus it remains to investigate the category $\operatorname{Add}(S \oplus I)$, which is equivalent to the category of projective right modules over $S^{\prime}=\operatorname{End}(S \oplus I)$. In the following lemma we show that this ring is semilocal.

Lemma 8.5. $S^{\prime}=\operatorname{End}(S \oplus I)$ is semilocal.

Proof. $I_{S}$ is a uniserial module, hence its Goldie dimension and its dual Goldie dimension is equal to 1 . Furthermore the Goldie dimension of $S_{S}$ is 1 , and the dual Goldie dimension of $S_{S}$ is 2 . It follows that the right $S$ module $S \oplus I$ has finite Goldie dimension, and finite dual Goldie dimension. Then its endomorphism ring is semilocal by [5, Thm. 4.3].

We need the following auxiliary lemma.

Lemma 8.6. $\operatorname{End}\left({ }_{S} K\right) \cong S$ canonically. Furthermore, $\operatorname{End}\left(I_{S}\right) \cong S$.

Proof. Since $K$ is an $S$-S-bimodule, $S$ acts on ${ }_{S} K$ by multiplication on the right. This action is faithful. Indeed, if $0 \neq h \in S$ and $f \in K \backslash I$, then (since $f$ is mono) $f h \neq 0$.

Thus $S$ is a subring of $\operatorname{End}\left({ }_{S} K\right)$. By Corollary 4.6, $\operatorname{ann}(S)(f)=S g$ for some $g \in I \backslash K$ such that $\operatorname{ann}(g)(S)=f S$.

Since $K=S f$, every $\alpha \in \operatorname{End}\left({ }_{S} K\right)$ is uniquely determined by $(f) \alpha \in K$. From $g f=0$ it follows that $g(f \alpha)=(g f) \alpha=0$. Thus $f \alpha \in \operatorname{ann}(g)(S)=f S$, hence $f \alpha=f h$ for some $h \in S$. Then $\alpha$ is a right multiplication by $h$, hence $\alpha \in S$.

The proof that $\operatorname{End}\left(I_{S}\right) \cong S$ is similar. 
Now we reveal a matrix structure in $S^{\prime}$.

Lemma 8.7. $S^{\prime}=\operatorname{End}\left(S_{S} \oplus I_{S}\right)$ is isomorphic to the ring $(\underset{I}{S} \underset{S}{S})$.

Proof. Clearly $\operatorname{End}(S \oplus I)$ is isomorphic to the $\operatorname{ring}\left(\begin{array}{l}\operatorname{Hom}(S, S) \operatorname{Hom}(I, S) \\ \operatorname{Hom}(S, I) \\ \operatorname{Hom}(I, I)\end{array}\right)$ acting on the column $\left(\begin{array}{c}S \\ I\end{array}\right)$ on the left. We have natural identifications: $\operatorname{Hom}(S, S)=S$, and $\operatorname{Hom}(S, I)=I$. By Lemma 8.6, we can identify $\operatorname{Hom}(I, I)$ with $S$. By similar arguments, every morphism from $I$ to $S$ is a left multiplication by $s \in S$, hence $\operatorname{Hom}(I, S)=S$.

It is readily checked that these identifications respect matrix multiplication.

Let $e_{1}=e_{11}, e_{2}=e_{22}$ be matrix units in $S^{\prime}$. In particular, $S^{\prime}=e_{1} S^{\prime} \oplus$ $e_{2} S^{\prime}$, hence $\mathrm{J}\left(S^{\prime}\right)=\mathrm{J}\left(e_{1} S^{\prime}\right) \oplus \mathrm{J}\left(e_{2} S^{\prime}\right)$. First we describe maximal submodules of $e_{i} S^{\prime}, i=1,2$. We use $J$ to denote $\mathrm{J}(S)=I \cap K$.

Proposition 8.8. 1) $(I, S)$ and $(K, K)$ (we omit the second zero row) are the only maximal submodules of $e_{1} S^{\prime}$. In particular, $J\left(e_{1} S^{\prime}\right)=(J, K)$.

2) $(I, I)$ and $(J, K)$ (we omit the first zero row) are the only maximal submodules of $e_{2} S^{\prime}$. In particular, $J\left(e_{2} S^{\prime}\right)=(J, J)$.

Therefore $\mathrm{J}\left(S^{\prime}\right)=\left(\begin{array}{cc}J & K \\ J & J\end{array}\right)$.

Proof. 1) If $(a, b) \in e_{1} S^{\prime}$, then $(a, b) S^{\prime}=(a S+b I, a S+b S)$. It is easily checked that $(I, S)$ and $(K, K)$ are submodules of $e_{1} S^{\prime}$.

If $a$ is invertible, that $(a, b) S^{\prime}=(S, S)=e_{1} S^{\prime}$, and if $a \in I$, then $(a, b) S^{\prime} \subseteq$ $(I, S)$. Thus we may assume that $a \in K \backslash I$.

If $b \in K$, then $(a, b) S^{\prime} \subseteq(K, K)$. Otherwise $b \notin K$, hence $b g \in I \backslash K(g$ is any element of $I \backslash K)$. It follows that $a+b g \in a S+b I$ is invertible, hence $a S+b I=S$ yields $(a, b) S^{\prime}=(S, S)$.

2) If $(i, b) \in e_{2} S^{\prime}$, then $(i, b) S^{\prime}=(i S+b I, i S+b S)$. It is easy to check that $(I, I)$ and $(\mathrm{J}, K)$ are submodules of $e_{2} S^{\prime}$.

If $b$ is invertible, then $(i, b) S^{\prime}=(I, S)=e_{2} S^{\prime}$, and if $b \in I$, then $(i, b) S^{\prime} \subseteq$ $(I, I)$. Thus we may assume that $b \in K \backslash I$.

If $i \in K$, then $(i, b) S^{\prime} \subseteq(K, K)$. Otherwise $i \in I \backslash K$, hence $i S+b I=$ $I+b I=I$. Also $i+b \in i S+b S$ is invertible, hence $i S+b S=S$. Thus $(a, b) S^{\prime}=(I, S)$ in this case.

From this we derive a description of simple $S^{\prime}$-modules.

Proposition 8.9. The following is a complete list of simple right $S^{\prime}$-modules:

1) $S_{1}=\left(\begin{array}{ll}S & S \\ 0 & 0\end{array}\right) /\left(\begin{array}{cc}I & S \\ 0 & 0\end{array}\right)$; 
2) $S_{2}=\left(\begin{array}{cc}S & S \\ 0 & 0\end{array}\right) /\left(\begin{array}{cc}K & K \\ 0 & 0\end{array}\right) \cong T_{2}=\left(\begin{array}{cc}0 & 0 \\ I & S\end{array}\right) /\left(\begin{array}{cc}0 & 0 \\ J & K\end{array}\right)$;

3) $T_{1}=\left(\begin{array}{ll}0 & 0 \\ I & S\end{array}\right) /\left(\begin{array}{ll}0 & 0 \\ I & I\end{array}\right)$.

Therefore $S^{\prime} / \mathrm{J}\left(S^{\prime}\right) \cong S / I \oplus S / I \oplus M_{2}(S / K)$.

Proof. By Lemma 8.5, $S^{\prime}$ is semilocal, hence $S^{\prime} / \mathrm{J}\left(S^{\prime}\right)$ is semisimple artinian. Since every simple $S^{\prime}$-module $N$ is annihilated by $\mathrm{J}\left(S^{\prime}\right)=\mathrm{J}\left(e_{1} S^{\prime}\right) \oplus \mathrm{J}\left(e_{2} S^{\prime}\right)$, it follows that $N$ is isomorphic to $e_{i} S^{\prime} / T$, where $T$ is a maximal submodules of $e_{i} S^{\prime}$. By Proposition 8.8 we obtain that $N$ is isomorphic to $S_{i}$ or $T_{i}$, $i=1,2$.

It is easily checked that $S_{2} \cong T_{2}$, but all the remaining simples are not isomorphic. Indeed, to prove that $S_{2} \cong T_{2}$ choose any $g \in I \backslash K$ and let $G=\left(\begin{array}{ll}0 & 0 \\ g & 0\end{array}\right)$. Since $G \cdot\left(\begin{array}{cc}K & K \\ 0 & 0\end{array}\right) \subseteq\left(\begin{array}{cc}0 & 0 \\ J & S\end{array}\right)$, the left multiplication by $G$ induces a morphism from $S_{2}$ to $T_{2}$. Since $G$ is not in $\left(\begin{array}{ll}0 & 0 \\ J & S\end{array}\right)$, this morphism is nonzero, hence an isomorphism.

Since $\operatorname{End}\left(S_{1}\right)=\operatorname{End}\left(T_{1}\right) \cong S / I$ and $\operatorname{End}\left(S_{2}\right)=\operatorname{End}\left(T_{2}\right) \cong S / K$, we obtain that $S^{\prime} / \mathrm{J}\left(S^{\prime}\right) \cong S / I \oplus S / I \oplus M_{2}(S / K)$.

Since $S^{\prime}$ is semilocal, the annihilators of simple $S^{\prime}$-modules are exactly the maximal two-sided ideals of $S^{\prime}$. In the following lemma we calculate them.

Lemma 8.10. $\operatorname{Ann}\left(S_{1}\right)=A, \operatorname{Ann}\left(T_{1}\right)=B$ and $\operatorname{Ann}\left(S_{2}\right)=\operatorname{Ann}\left(T_{2}\right)=C$, where $A, B$ and $C$ are the following ideals:

$$
A=\left(\begin{array}{cc}
I & S \\
I & S
\end{array}\right), \quad B=\left(\begin{array}{cc}
S & S \\
I & I
\end{array}\right), \quad \text { and } \quad C=\left(\begin{array}{cc}
K & K \\
J & K
\end{array}\right) .
$$

Thus $A, B$ and $C$ form a complete list of two-sided maximal ideal of $S^{\prime}$, hence $\mathrm{J}\left(S^{\prime}\right)=A \cap B \cap C$.

Proof. By inspection.

Note that, apart of finitely presented modules, by Lemma 6.4 we know (at least if $\mathrm{J}(R)_{R}$ is countably generated) that $K_{S}$ is a projective, hence pureprojective module. The following remark shows that $\mathrm{J}_{S}$ is pure-projective.

Remark 8.11. $S_{S} \oplus \mathrm{J}_{S} \cong I_{S} \oplus K_{S}$.

Proof. Since $I+K=S$ and $S$ is projective, the short exact sequence $0 \rightarrow$ $\mathrm{J}=I \cap K \rightarrow I \oplus K \rightarrow S \rightarrow 0$ splits. It follows that $\mathrm{J}_{S}$ is a direct summand of the pure-projective module $I \oplus K$, hence pure-projective.

Now we calculate the invariants of pure-projective right $S$-modules from $\operatorname{Add}(S \oplus I)$. Recall that there is an equivalence between this category, and 
the category of projective right $S^{\prime}$-modules, given by the functor $\operatorname{Hom}(S \oplus$ $I,-)$.

Let $P$ be a projective right $S^{\prime}$-module. Define $\alpha(P)$ to be the dimension of the vector space $P / P A$ over the skew field $S^{\prime} / A \cong S / I$. Note that this is just the multiplicity of the simple $S^{\prime}$-module $S_{1}$ in a (homogeneous semisimple) module $P / P A$.

Similarly, let $\beta(P)$ denote the dimension of the vector space $P / P B$ over the skew field $S^{\prime} / B \cong S / I$. This is equal to the multiplicity of the simple $S^{\prime}$-module $T_{1}$ in a (homogeneous semisimple) module $P / P B$.

Finally, let us consider the module $P / P C$ over the (semisimple artinian) ring $S^{\prime} / C=M_{2}(S / K)$. Then $P / P C$ is a direct sum of copies of the unique simple $M_{2}(S / K)$ module $S_{2}$. Let $\gamma(P)$ be the multiplicity of this simple in $P / P C$.

Thus the dimension of $P, \operatorname{dim}(P)$, is a triple of cardinals $(\alpha(P), \beta(P), \gamma(P))$.

Now we are in a position to calculate the dimensions of known indecomposable pure-projective $S$-modules. We we will use the same letter to denote a pure-projective right $S$-module $N$, and its image $\operatorname{Hom}(S \oplus I, N)$ which is a projective right $S^{\prime}$-module.

Proposition 8.12. 1) $\operatorname{dim}(S)=(1,0,1)$;

2) $\operatorname{dim}(K)=(1,0,0)$;

3) $\operatorname{dim}(I)=(0,1,1)$;

4) $\operatorname{dim}(\mathrm{J})=(0,1,0)$.

Proof. Note that $S$ goes to $e_{1} S^{\prime}=\left(\begin{array}{cc}S & S \\ 0 & 0\end{array}\right)$ via the above equivalence.

The remaining part of the proof is just matrix calculations.

1) $e_{1} S^{\prime} \cdot A=\left(\begin{array}{cc}S & S \\ 0 & 0\end{array}\right) \cdot\left(\begin{array}{cc}I & S \\ I & S\end{array}\right)=\left(\begin{array}{ll}I & S \\ 0 & 0\end{array}\right)$, hence $\alpha(S)=1$.

$e_{1} S^{\prime} \cdot B=\left(\begin{array}{cc}S & S \\ 0 & 0\end{array}\right) \cdot\left(\begin{array}{cc}S & S \\ I & I\end{array}\right)=\left(\begin{array}{cc}S & S \\ 0 & 0\end{array}\right)$, hence $\beta(S)=0$.

$e_{1} S^{\prime} \cdot C=\left(\begin{array}{cc}S & S \\ 0 & 0\end{array}\right) \cdot\left(\begin{array}{cc}K & K \\ J & K\end{array}\right)=\left(\begin{array}{cc}K & K \\ 0 & 0\end{array}\right)$. Since $e_{1} S^{\prime} / e_{1} S^{\prime} C=S_{2}$ is a simple module, therefore $\gamma(S)=1$.

Thus $\operatorname{dim}(S)=(1,0,1)$.

2) Note that $K$ goes to $\operatorname{Hom}(S \oplus I, K)$, which can be identified with $\left(\begin{array}{cc}K & K \\ 0 & 0\end{array}\right)$

$K \cdot A=\left(\begin{array}{cc}K & K \\ 0 & 0\end{array}\right) \cdot\left(\begin{array}{cc}I & S \\ I & S\end{array}\right)=\left(\begin{array}{cc}J & K \\ 0 & 0\end{array}\right)$, hence $\alpha(K)=1$ (see Lemma 5.4 for the equality $K I=J)$.

$K \cdot B=\left(\begin{array}{cc}K & K \\ 0 & 0\end{array}\right) \cdot\left(\begin{array}{cc}S & S \\ I & I\end{array}\right)=\left(\begin{array}{cc}K & K \\ 0 & 0\end{array}\right)$, hence $\beta(K)=0$.

$K \cdot C=\left(\begin{array}{cc}K & K \\ 0 & 0\end{array}\right) \cdot\left(\begin{array}{cc}K & K \\ J & K\end{array}\right)=\left(\begin{array}{cc}K & K \\ 0 & 0\end{array}\right)$, hence $\gamma(K)=0$ (since $K$ is idempotent: see a remark after Lemma 6.4).

Thus $\operatorname{dim}(K)=(1,0,0)$. 
To make the above identification more transparent, note that $\left(\begin{array}{cc}K & K \\ 0 & 0\end{array}\right)$ is a projective module. Indeed, let $f_{i} \in K \backslash I, f_{i+1} f_{i}=f_{i}$ be a sequence of generators of $K_{S}$ (see Lemma 6.4). Then $f_{i}^{\prime}=\left(\begin{array}{cc}f_{i} & 0 \\ 0 & 0\end{array}\right)$ is a sequence of generators for $\left(\begin{array}{cc}K & K \\ 0 & 0\end{array}\right)$ such that $f_{i+1}^{\prime} f_{i}^{\prime}=f_{i}^{\prime}$, hence this module is projective.

3) $I$ goes to $e_{2} S^{\prime}=\left(\begin{array}{ll}0 & 0 \\ I & S\end{array}\right)$.

$I \cdot A=\left(\begin{array}{cc}0 & 0 \\ I & S\end{array}\right) \cdot\left(\begin{array}{ll}I & S \\ I & S\end{array}\right)=\left(\begin{array}{cc}0 & 0 \\ I & S\end{array}\right)$, hence $\alpha(I)=0$ (since $I$ is idempotent).

$I \cdot B=\left(\begin{array}{cc}0 & 0 \\ I & S\end{array}\right) \cdot\left(\begin{array}{cc}S & S \\ I & I\end{array}\right)=\left(\begin{array}{ll}0 & 0 \\ I & I\end{array}\right)$, hence $\beta(I)=1$.

$I \cdot C=\left(\begin{array}{cc}0 & 0 \\ I & S\end{array}\right) \cdot\left(\begin{array}{cc}K & K \\ J & K\end{array}\right)=\left(\begin{array}{ll}0 & 0 \\ J & K\end{array}\right)$. Since $e_{2} S^{\prime} / e_{2} S^{\prime} C \cong T_{2}$ is a simple $S^{\prime}$-module, we conclude that $\gamma(I)=1$.

Thus $\operatorname{dim}(I)=(0,1,1)$.

4) J goes to $\operatorname{Hom}(S \oplus I, \mathrm{~J})$ which can be identified with $\left(\begin{array}{ll}0 & 0 \\ J & K\end{array}\right)$.

$\mathrm{J} \cdot A=\left(\begin{array}{cc}0 & 0 \\ J & K\end{array}\right) \cdot\left(\begin{array}{cc}I & S \\ I & S\end{array}\right)=\left(\begin{array}{cc}0 & 0 \\ J & K\end{array}\right)$, hence $\alpha(\mathrm{J})=0$.

$\mathrm{J} \cdot B=\left(\begin{array}{cc}0 & 0 \\ J & K\end{array}\right) \cdot\left(\begin{array}{cc}S & S \\ I & I\end{array}\right)=\left(\begin{array}{ll}0 & 0 \\ J & J\end{array}\right)$, hence $\beta(\mathrm{J})=1$.

$\mathrm{J} \cdot C=\left(\begin{array}{cc}0 & 0 \\ J & K\end{array}\right) \cdot\left(\begin{array}{cc}K & K \\ J & K\end{array}\right)=\left(\begin{array}{cc}0 & 0 \\ J & K\end{array}\right)$, hence $\gamma(\mathrm{J})=0$.

Thus $\operatorname{dim}(\mathrm{J})=(0,1,0)$.

Concerning the above identification, note that $\left(\begin{array}{ll}0 & 0 \\ J & K\end{array}\right)$ is a projective right $S^{\prime}$-module. Indeed, choose generators $f_{i}$ for $K_{S}$ as in 2$)$. Then $f_{i}^{\prime \prime}=\left(\begin{array}{ll}0 & 0 \\ 0 & f_{i}\end{array}\right)$ are generators for $\left(\begin{array}{cc}0 & 0 \\ J & K\end{array}\right)$ such that $f_{i+1}^{\prime \prime} f_{i}^{\prime \prime}=f_{i}^{\prime \prime}$, hence this module is projective.

Note that, by Proposition 8.12, $\operatorname{dim}(S \oplus \mathrm{J})=(1,1,1)=\operatorname{dim}(I \oplus K)$, so Lemma 8.11 is nothing surprising.

We observe some properties of the two-sided ideal $C$.

Remark 8.13. $C=\left(\begin{array}{cc}K & K \\ J & K\end{array}\right)$ is principal as a left ideal of $S^{\prime}$. Furthermore $C$ is projective as a right $S^{\prime}$-module.

Proof. We prove that, if $f \in K \backslash I$, then $s=\left(\begin{array}{ll}f & 0 \\ 0 & f\end{array}\right)$ generates $C$. Indeed

$$
S^{\prime} s=\left(\begin{array}{cc}
S & S \\
I & S
\end{array}\right) \cdot\left(\begin{array}{cc}
f & 0 \\
0 & f
\end{array}\right)=\left(\begin{array}{cc}
S f & S f \\
I f & S f
\end{array}\right)=\left(\begin{array}{cc}
K & K \\
J & K
\end{array}\right)=C .
$$

Note that $C_{S^{\prime}}$ is a direct sum of projective right $S^{\prime}$-modules: $\left(\begin{array}{cc}K & K \\ 0 & 0\end{array}\right)$ (the image of $K)$, and $\left(\begin{array}{ll}0 & 0 \\ J & K\end{array}\right)$ (the image of $\mathrm{J}$ ). Thus, $C$ is a projective right $S^{\prime}$-module.

Theorem 8.14. Let $P$ be a pure-projective right $S$-module. Then $P$ is isomorphic to a direct sum of copies of $S_{S}, K_{S}, I_{S}, \mathrm{~J}_{S}$, and $S / I_{S}$.

We prove this theorem as a sequence of subsequent reductions. First, by Corollary 8.4, $P$ is a direct sum of a pure projective module $P^{\prime} \in \operatorname{Add}(S \oplus I)$ and copies of $S / I$. 
Therefore, from the very beginning we may assume that $P \in \operatorname{Add}(S \oplus I)$. Recall that the category $\operatorname{Add}(S \oplus I)$ is equivalent to the category of right projective $S^{\prime}$-modules. Thus, in this section we keep standard identifications (see Section 8 before Proposition 8.12) between pure projective right $S$ modules, and projective right $S^{\prime}$-modules. For instance, $S_{S}$ corresponds to $e_{1} S^{\prime}$, and $I_{S}$ corresponds to $e_{2} S^{\prime}$.

In particular, we may consider $P$ as a projective $S^{\prime}$-module.

By Kaplansky's theorem we may assume that $P$ is countably generated, hence $\alpha(P), \beta(P), \gamma(P) \leq \omega$. First we eliminate the case when $\alpha(P)=$ $\beta(P)=\gamma(P)=\omega$. Indeed, by Proposition 8.12, $\operatorname{dim}\left(e_{1} S^{\prime}\right)=\operatorname{dim}(S)=$ $(1,0,0)$ and $\operatorname{dim}\left(e_{2} S^{\prime}\right)=\operatorname{dim}(I)=(0,1,1)$. It follows that $\operatorname{dim}\left(S^{\prime(\omega)}\right)=$ $(\omega, \omega, \omega)$, hence $P \cong S^{\prime(\omega)}$ by Corollary 7.2 .

Otherwise we may assume that one of the dimensions, $\alpha(P), \beta(P)$, or $\gamma(P)$, is finite. Now we reduce a classification to the case, when one of coordinates is zero.

Lemma 8.15. Let $P$ be a projective right $S^{\prime}$ module such that one component of $\operatorname{dim}(P)$ is finite. Then $P=P^{\prime} \oplus Q$, where $Q$ is a finite direct sum of modules $e_{1} S^{\prime}$ and $e_{2} S^{\prime}$, and one of coordinates of $\operatorname{dim}\left(P^{\prime}\right)$ is zero.

Proof. Note that (see Lemma 8.12) $\operatorname{dim}\left(e_{1} S^{\prime}\right)=(1,0,1)$, hence $e_{1} S^{\prime}$ is a projective cover of $S_{1} \oplus S_{2}$. Furthermore, $\operatorname{dim}\left(e_{2} S^{\prime}\right)=(0,1,1)$, hence $e_{2} S^{\prime}$ is a projective cover of $S_{2} \oplus T_{1}=T_{2} \oplus T_{1}$.

Suppose that $\alpha(P)=k>0$ is a minimal finite number among $\alpha(P), \beta(P)$, and $\gamma(P)$. Using projective covers, it is easily seen that $P=P^{\prime} \oplus e_{1} S^{\prime k}$, hence (calculating dimensions) $\alpha\left(P^{\prime}\right)=0$.

Similarly, if $\beta(P)=k>0$ is minimal finite number between $\alpha(P), \beta(P)$, and $\gamma(P)$, then $P=P^{\prime} \oplus e_{2} S^{\prime k}$, where $\beta\left(P^{\prime}\right)=0$.

Finally, if $\gamma(P)>0$ is minimal and finite, then we can use either $e_{1} S^{\prime}$ or $e_{2} S^{\prime}$ to guarantee $\gamma\left(P^{\prime}\right)=0$.

Thus we may assume that one of the coordinates, $\alpha(P), \beta(P)$, or $\gamma(P)$ is zero.

Below we give an easy structure theory for $P$ in the case when $\alpha(P)=0$, or $\beta(P)=0$. For the following lemma note that any countably generated pure right ideal of any ring is projective.

Lemma 8.16. Let $P$ be a pure right ideal of a ring $T$, and let $Q$ be a projective right $T$-module. Then $Q \in \operatorname{Add}(P)$ if and only if $Q=Q P$. 
Proof. Suppose that $Q \in \operatorname{Add}(P)$, and take any $m \in Q$. Then there is a morphism $f=\sum_{i} f_{i}: P^{k} \rightarrow Q$ such that $m \in \operatorname{im}(f)$, i.e. $\sum_{i} f_{i}\left(r_{i}\right)=m$ for some $r_{i} \in P$. Since $P$ is pure in $T_{T}$, there are $s_{i} \in P$ such that $s_{i} r_{i}=r_{i}$. Then $m=\sum_{i} f_{i}\left(r_{i}\right)=\sum_{i} f_{i}\left(s_{i}\right) r_{i} \in Q P$.

For the converse, let $Q=Q P$. It suffices to prove that any $m \in Q$ is in the image of a map $f=\sum_{i} f_{i}: P^{k} \rightarrow Q$. From $m \in Q P$ we conclude that $m=\sum_{i} m_{i} r_{i}$, where $m_{i} \in Q$, and $r_{i} \in P$. We define $g_{i}: T \rightarrow Q$ sending 1 to $m_{i}$. Let $f_{i}$ be the restriction of $g_{i}$ on $P$. If $f=\sum_{i} f_{i}: P^{k} \rightarrow Q$, then $f\left(\sum_{i} r_{i}\right)=\sum_{i} g_{i}(1) r_{i}=\sum_{i} m_{i} r_{i}=m$.

Remark 8.17. Note that $Q=Q P$ in Lemma 8.16 can be replaced by $Q=$ $Q P^{\prime}$, where $P^{\prime}=R P$ is a two-sided ideal generated by $P$.

Now we are ready to classify projective right $S^{\prime}$-modules $P$ with $\alpha(P)=0$ or $\beta(P)=0$.

Proposition 8.18. Let $P$ be a projective right $S^{\prime}$-module.

1) If $\beta(P)=0$, then $P \in \operatorname{Add}\left(e_{1} S^{\prime}\right)$, hence $P \cong e_{1} S^{(\lambda)} \oplus K^{(\mu)}$ for some $\lambda$ and $\mu$.

2) If $\alpha(P)=0$, then $P \in \operatorname{Add}\left(e_{2} S^{\prime}\right)$, hence $P \cong e_{2} S^{(\lambda)} \oplus \mathrm{J}^{(\mu)}$.

Proof. 1) $\beta(P)=0$ means that $P=P B$. To prove $P \in \operatorname{Add}\left(e_{1} S^{\prime}\right)$, by Remark 8.17 it suffices to check that $S^{\prime} e_{1} S^{\prime}=B$. Indeed

$$
S^{\prime} e_{1} S^{\prime}=\left(\begin{array}{cc}
S & S \\
I & S
\end{array}\right) \cdot\left(\begin{array}{cc}
S & S \\
0 & 0
\end{array}\right)=\left(\begin{array}{cc}
S & S \\
I & I
\end{array}\right)=B .
$$

Now the category $\operatorname{Add}\left(e_{1} S^{\prime}\right)$ is equivalent to the category of projective right $\operatorname{End}\left(e_{1} S^{\prime}\right)$-modules, and $\operatorname{End}\left(e_{1} S^{\prime}\right)=e_{1} S^{\prime} e_{1}=S$.

By Theorem 7.3, every right projective $S$-module is of the form $S^{(\lambda)} \oplus$ $K^{(\mu)}$. Now $S$ goes to $e_{1} S^{\prime}$ via the above equivalence, and we use $K$ to denote the image of $K$.

2) $\alpha(P)=0$ means $P=P A$. To prove $P \in \operatorname{Add}\left(e_{2} S^{\prime}\right)$, by Remark 8.17 it suffices to check that $S^{\prime} e_{2} S^{\prime}=A$. Indeed,

$$
S^{\prime} e_{2} S^{\prime}=\left(\begin{array}{cc}
S & S \\
I & S
\end{array}\right) \cdot\left(\begin{array}{ll}
0 & 0 \\
I & S
\end{array}\right)=\left(\begin{array}{ll}
I & S \\
I & S
\end{array}\right)=A .
$$

Thus $P \in \operatorname{Add}\left(e_{2} S^{\prime}\right)$. Now the category $\operatorname{Add}\left(e_{2} S^{\prime}\right)$ is equivalent to the category of projective right $\operatorname{End}\left(e_{2} S^{\prime}\right)$-modules, and $\operatorname{End}\left(e_{2} S^{\prime}\right)=e_{2} S^{\prime} e_{2}=$ $S$. 
By Theorem 7.3, every right projective $S$-module is of the form $S^{(\lambda)} \oplus$ $K^{(\mu)}$. Now $S$ corresponds to $e_{2} S^{\prime}$ (i.e., $I$ ) via the above equivalence, and $K$ corresponds to J.

Thus it remains to consider the case $\operatorname{dim}(P)=(\alpha, \beta, 0)$, where $0<\alpha, \beta \leq$ $\omega$. By Proposition 8.12, $\operatorname{dim}(K)=(1,0,0)$ and $\operatorname{dim}(J)=(0,1,0)$, hence $\operatorname{dim}\left(K^{(\alpha)} \oplus J^{(\beta)}\right)=(\alpha, \beta, 0)$. By Corollary 7.2 we obtain $P \cong K^{(\alpha)} \oplus \mathrm{J}^{(\beta)}$, as desired.

Now we are in a position to describe the projective spectrum of $S^{\prime}$.

Proposition 8.19. The projective spectrum of $S^{\prime}$ consists of vectors $(\alpha+$ $\beta, \gamma+\delta, \alpha+\gamma)$. Furthermore, the finite projective spectrum of $S^{\prime}$ consists of vectors $(n, m, n+m)$, and the infinite projective spectrum of $S^{\prime}$ consists of vectors $(\beta, \delta, 0)$.

Proof. Indeed, from the proof of Theorem 8.14 it follows that every projective $S^{\prime}$-module is isomorphic to the module $S^{(\alpha)} \oplus K^{(\beta)} \oplus I^{(\gamma)} \oplus J^{(\delta)}$. By Proposition 8.12 this module has dimension vector $(\alpha+\beta, \gamma+\delta, \alpha+\gamma)$.

Furthermore, every finitely generated projective $S^{\prime}$-module is isomorphic to $S^{n} \oplus I^{m}$, hence has $(n, m, n+m)$ as a dimension vector. 


\section{REFERENCES}

[1] M. Auslander, I. Reiten, S. Smalø, Representation Theory of Artin Algebras, Vol. 40, Cambridge Studies in Mathematics, Cambridge University Press, 1995.

[2] H.H. Brungs, N.I. Dubrovin, A classification and examples of rank one chain domains, Trans. Amer. Math. Soc., 355 (2003), 2733-2753.

[3] P.M. Cohn, Unique factorization domains, Amer. Math. Monthly, 80 (1973), 1-17.

[4] N. Dubrovin, Chain domains, Vestnik Moskov. Univ. Ser. I Mat. Mekh., 2 (1980), $51-54$.

[5] A. Facchini, Module Theory: Endomorphism Rings and Direct Sum Decompositions in Some Classes of Modules, Progress in Mathematics, Vol. 167, Birkhäuser, 1998.

[6] A. Facchini, D. Herbera, $K_{0}$ of a semilocal ring, J. Algebra, 225 (2000), 47-69.

[7] A. Facchini, D. Herbera, I. Sakhaev, Finitely generated flat modules and a characterization of semiperfect rings, Comm. Algebra, 31(9) (2003), 4195-4214.

[8] V. Gerasimov, I. Sakhaev, A counterexample to two conjectures on projective and flat modules, Sibirsk. Mat. Zh., 25(6) (1984), 31-35.

[9] Y. Hinohara, Projective modules over weakly noetherian rings, J. Math. Soc. Japan, 15(1) (1963), 75-88.

[10] C.U. Jensen, On homological dimension of rings with countably generated ideals, Math. Scand., 18 (1966), 97-105.

[11] C.U. Jensen, H. Lenzing, Model Theoretic Algebra, Algebra, Logic and Application Series, Vol. 2, Gordon and Breach, 1989.

[12] T.Y. Lam, A First Course in Noncommutative Rings, 2nd edition, Graduate Texts in Mathematics, Vol. 131, Springer, 1991.

[13] T.Y. Lam, Lectures on Modules and Rings, Graduate Texts in Mathematics, Vol. 199, Springer, 1999.

[14] J.C. McConell, J.C. Robson, Noncommutative Noetherian Rings, Graduate Studies in Mathematics, Vol. 30, American Math. Soc., 2001.

[15] W. McGovern, G. Puninski, Ph. Rothmaler, When every projective module is a direct sum of finitely generated modules, Preprint, 2006.

[16] M. Prest, Model Theory and Modules, London Math. Soc. Lecture Note Series, Vol. 130, Cambridge University Press, 1987.

[17] P. Příhoda, Projective modules are determined by their radical factors, Preprint, 2006.

[18] G. Puninski, Serial Rings, Kluwer Academic Publishers, 2001.

[19] G. Puninski, Some model theory over a nearly simple uniserial domain and decompositions of serial modules, J. Pure Appl. Algebra, 163 (2001), 319-337.

[20] G. Puninski, Projective modules over the endomorphism ring of a biuniform module, J. Pure Appl. Algebra, 188 (2004), 227-246.

[21] R. Wisbauer, Foundations of Module and Ring Theory, Algebra, Logic and Application Series, Vol. 3, Gordon and Breach, 1991. 
(N. Dubrovin) Department of Mathematics, Vladimir State University, Gorky Street 87, Vladimir, 600000, Russia

E-mail address: ndubrovin@rumbler.ru

(G. Puninski) School of Mathematics, Manchester University, Lamb BuildInG, Booth Street East, Manchester, M13 9PL, UK

E-mail address: gpuninski@maths.man.ac.uk 